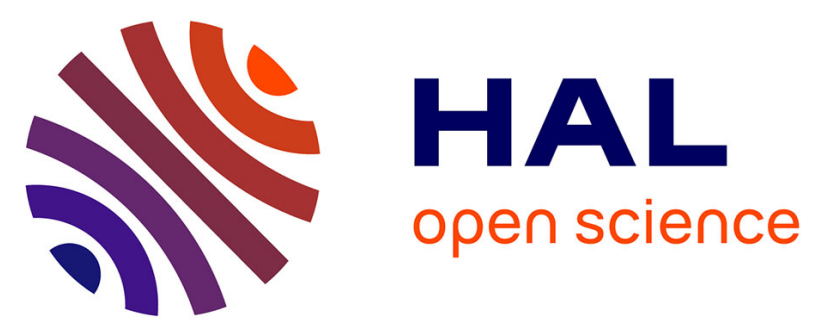

\title{
Utilisation de critères métaboliques et morphogénétiques pour l'analyse de la diapause de populations naturelles d'Ostrinia nubilalis Hbn. (Lep., Pyralidae)
}

Christophe Gadenne, Lionel Peypelut, Lucien Lavenseau, Brigitte Got

\section{To cite this version:}

Christophe Gadenne, Lionel Peypelut, Lucien Lavenseau, Brigitte Got. Utilisation de critères métaboliques et morphogénétiques pour l'analyse de la diapause de populations naturelles d'Ostrinia nubilalis Hbn. (Lep., Pyralidae). Agronomie, 1987, 7 (10), pp.769-777. hal-00884952

\section{HAL Id: hal-00884952 https://hal.science/hal-00884952}

Submitted on 1 Jan 1987

HAL is a multi-disciplinary open access archive for the deposit and dissemination of scientific research documents, whether they are published or not. The documents may come from teaching and research institutions in France or abroad, or from public or private research centers.
L'archive ouverte pluridisciplinaire HAL, est destinée au dépôt et à la diffusion de documents scientifiques de niveau recherche, publiés ou non, émanant des établissements d'enseignement et de recherche français ou étrangers, des laboratoires publics ou privés. 


\title{
Utilisation de critères métaboliques et morpho- génétiques pour l'analyse de la diapause de populations naturelles d'Ostrinia nubilalis $\mathrm{Hbn}$. (Lep., Pyralidae)
}

Christophe GADENNE, Lionel PEYPELUT, Lucien LAVENSEAU \& Brigitte GOT*

Université de Bordeaux I, Laboratoire de Neuroendocrinologie, UA C.N.R.S. 1138, avenue des Facultés, F 33405 Talence Cedex

* I.N.R.A., Station de Zoologie, route de St-Cyr, F 78000 Versailles

\begin{abstract}
Des populations de pyrale du maïs de plein champ, issues d'infestations artificielles réalisées tout au long du mois de juillet, ont été étudiées en 1984 dans les stations I.N.R.A. de Colmar et Versailles et en 1985 et 1986 à Versailles uniquement. L'utilisation de critères physiologiques tels que la tréhalosémie et l'observation des disques imaginaux alaires permet de mieux comprendre les différentes étapes caractérisant leur diapause. L'entrée apparente et la sortie de diapause s'effectuent au même moment quelles que soient la date d'infestation des pieds de maïs et l'origine géographique des pyrales. La tréhalosémie, élevée pendant l'hiver, chute au mois d'avril, soit 2 mois avant l'apparition d'un taux de $50 \mathrm{p}$. 100 de nymphes dans les prélèvements et 1 mois et demi avant la reprise du développement des ébauches alaires qui a lieu au mois de mai. A ce moment-là, peuvent être mises en évidence des périodes de quiescence. Provoquées par des conditions climatiques momentanément défavorables, elles sont caractérisées par une forte tréhalosémie et un arrêt de développement des ébauches alaires. L'étude du glycérol est un bon critère supplémentaire de levée de diapause, sa baisse de concentration étant alors irréversible.

Malgré leurs imperfections, l'utilisation de ces critères, d'emploi très simple et nécessitant peu de matériel, $s$ 'avère déjà très utile dans le suivi des populations du ravageur.
\end{abstract}

Mots clés additionnels : Tréhalose, glycérol, disques imaginaux alaires. Hbn. (Lep., Pyralidae).

\begin{abstract}
Natural strains of the European corn borer were obtained from egg infestations in July 1984 (I.N.R.A. research centers of Versailles and Colmar) and in 1985-1986 (Versailles only). Characterization of diapause was greatly improved by the use of physiological criteria such as trehalosemia and the study of imaginal wing discs. Presumptive diapause onset and diapause termination were independant of the date of oviposition on corn plants and of the geographic origin of the strains. Trehalosemia, high in winter, decreased in April, 2 months before the $50 \%$ pupation rate of the samples and 6 weeks before resumption of wing disc development in May. A quiescence phase, due to temporary bad climatic conditions, can occur in May. It is characterized by a high trehalosemia and an arrest of development of the wing discs. During diapause termination, the study of glycerol titre was a good additional criterion, for its concentration decrease was found to be irreversible. Although imperfect, these criteria, easy to handle and needing little material, are already in use to follow the population evolution of the pest.
\end{abstract}

Additional key words : Trehalose, glycerol, imaginal wing discs. 


\section{INTRODUCTION}

La larve d'Ostrinia nubilalis provoque d'importants dégâts dans les cultures de maïs, particulièrement dans le centre de la France, et sa zone d'incidence économique est en constante expansion vers le Nord-Ouest. Les méthodes de prévision de lutte sont donc plus que jamais d'actualité. Au début de l'hiver, la pyrale de dernier stade larvaire subit une diapause qui dure de 8 à 9 mois, jusqu'au printemps. Beaucoup d'études ont été menées, en laboratoire notamment, sur la levée de diapause, en utilisant comme preuve essentielle de la reprise du développement l'apparition des premières nymphes (BECK \& HANEC, 1960). C'est également ce critère tardif qui est utilisé, seul, pour les méthodes actuelles de prévision d'apparition des adultes. D'autre part, MCLEOD (1976) et STENGEL (1982) ont essayé de prévoir la date d'infestation à partir d'un modèle de degrés-jours. Les sommes de degrés-jours ainsi calculées se sont révélées dépendantes du lieu géographique, et parfois très variables en un même lieu (HAWLITZKY, 1986). L'élaboration de modèles de prévision plus performants est actuellement limitée par la méconnaissance des mécanismes de sortie de diapause.

Pour tenter d'analyser avec plus de précision le phénomène de diapause chez la pyrale du maïs, nous utilisons dans notre équipe divers critères physiologiques (LAVEnseau \& Trabelsi, 1983). Deux d'entre eux sont exploités ici : un critère métabolique, la tréhalosémie, et un critère morphogénétique, le développement des ébauches alaires. Chez les larves de pyrale en diapause, la tréhalosémie est importante. Elle chute ensuite au moment de la reprise du développement pour redevenir comparable à celle des larves élevées en développement continu. Ceci prolonge les résultats de ASAHINA \& TANNO (1964) sur Trichocampus populi Okamoto, de MOREAU (1969) sur Pieris brassicae L., et HAYAKawa \& CHINo (1981) sur Philosamia cynthia Drury. Pendant la diapause, la morphologie des ébauches alaires ne dépasse pas un stade peu avancé, que nous définirons. Développement et différenciation reprennent après la rupture de diapause (LAVENSEAU \& T'RABELSI, 1983). L'analyse de ces critères nous permet donc de distinguer les larves en diapause des larves en développement continu ou ayant rompu leur diapause.

L'objectif de ce travail était d'éprouver la fiabilité de ces méthodes, mises au point en laboratoire, sur des populations de pyrales de plein champ et de tester leur utilisation dans un modèle de prévision des dates d'infestation.

L'expérimentation a porté sur 3 années. Au printemps 1984, nous avons étudié 2 populations géographiquement distinctes, une dans la région de Colmar et une à Versailles. Au cours des 2 années suivantes (19841985 et 1985-1986), les critères physiologiques ont été testés à Versailles seulement à partir d'infestations artificielles pratiquées à différentes dates de la période naturelle de vol.

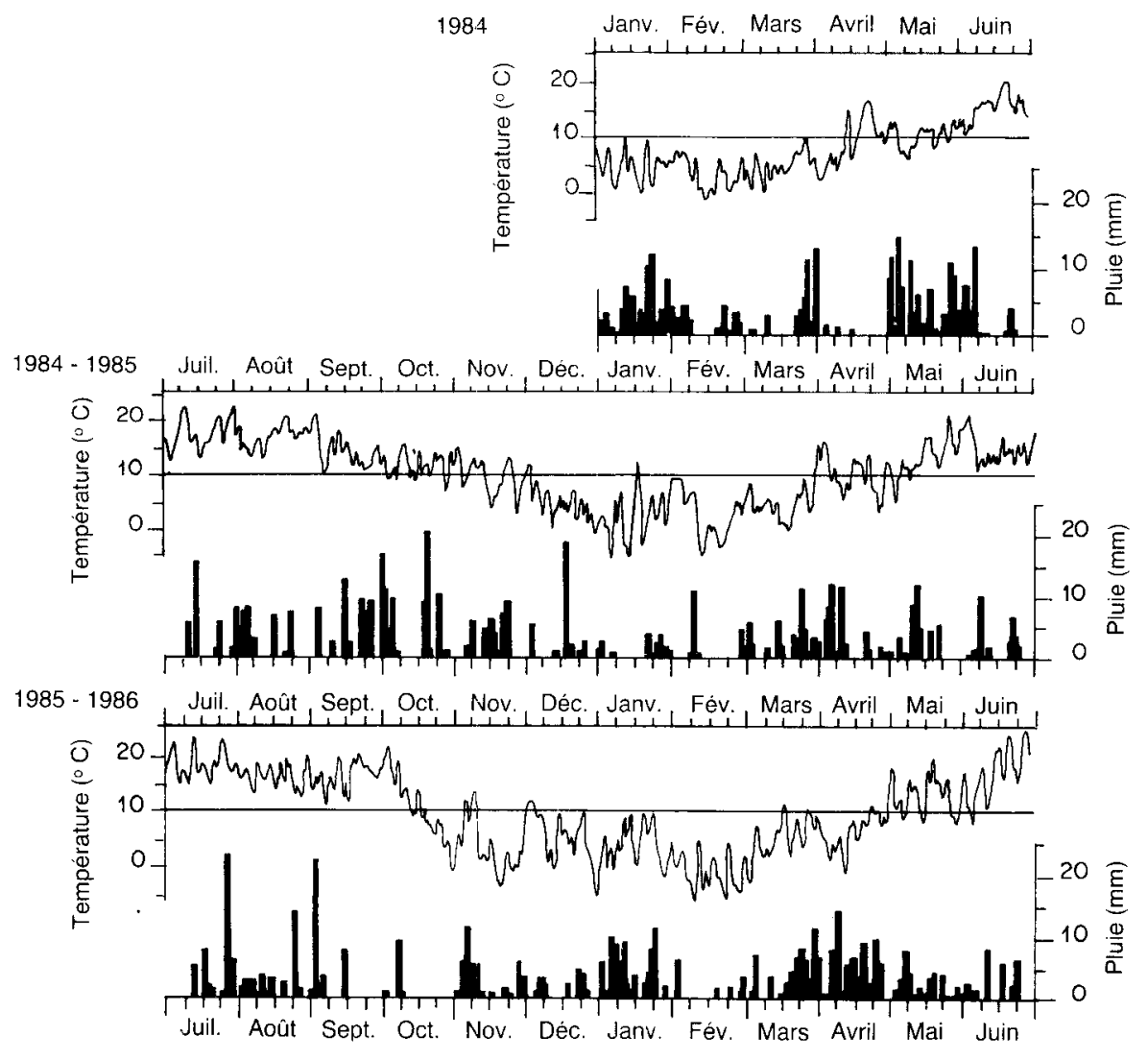

Figure 1

Evolution de la température moyenne journalière (courbes continues) et des précipitations (histogrammes) à Versailles, pour les saisons d'expérimentation de 1984, 1984-1985, 1985-1986. Le seuil de $10{ }^{\circ} \mathrm{C}$, utilisé couramment comme base de degrés-jours pour la pyrale, est repéré sur les graphiques de températures.
Variation in daily mean temperature (continuous lines) and rain (boxes) at Versailles for field trials in $1984 ; 1984-1985,1985-1986$. The $10{ }^{\circ} \mathrm{C}$ degree-day base currently used for the European corn borer is shown on the temperature graphs. 


\section{MATÉRIEL ET MÉTHODES}

\section{A. Protocole expérimental}

Pour apprécier le comportement de nos critères sur des populations différentes, nous les avons appliqués à des larves strictement monovoltines provenant de la station I.N.R.A. de Colmar (Alsace), et à des larves à tendance polyvoltine de la station I.N.R.A. de Versailles (Ile-de-France). Les larves en diapause, récoltées pendant tout le printemps 1984, provenaient de plants de maïs préalablement infestés avec des pontes l'été précédent, et conservés sous abri. Dix pyrales étaient envoyées, vivantes, à Bordeaux, chaque semaine pour analyse.

Pour apprécier l'influence de la date de ponte sur la date de sortie de diapause, nous avons profité d'une expérimentation menée à Versailles au domaine I.N.R.A. de la Verrière (GOT, 1985). Afin de multiplier les séquences climatiques étudiées, des infestations artificielles ont été réalisées à 4 dates différentes (3, 10, 17 et 24 juillet 1984$)$, avec des pontes de pyrale âgées de moins de 2 jours, obtenues à partir d'élevage à la station I.N.R.A. du Magneraud. Le développement des larves issues d'infestations artificielles étant supposé homogène, les prélèvements étaient effectués systématiquement, sur les rangs successifs des différentes parcelles. La température et les précipitations ont été enregistrées sur une centrale d'acquisition automatique (fig. 1). Dès l'observation des premières larves du dernier stade, 15 chenilles par lot ont été prélevées dans la zone centrale de chaque parcelle, et expédiées, vivantes, à Bordeaux. Les envois ont été réalisés chaque semaine jusqu'à l'installation confirmée de la diapause, puis une fois par mois pendant l'hiver, et de nouveau chaque semaine à partir du mois d'avril, date présumée de la rupture de diapause (fig. 2). Durant toute cette période, les cannes de maïs infestées ont été conservées sur pied dans le champ.

L'opération fut renouvelée, en juillet 1985, mais avec seulement 2 infestations artificielles pratiquées à 4 semaines d'intervalle : l'une le 4 juillet, correspondant au début de l'apparition des adultes, l'autre le $1^{\text {er }}$ août, correspondant à la fin de la période de vol des adultes.

\section{B. Dosage du tréhalose et du glycérol}

La chromatographie gazeuse quantitative a été utilisée. A $20 \mu \mathrm{l}$ d'hémolymphe (soit le sang de 1 à 2 chenilles), prélevés en présence de phénylthiourée pour éviter toute dégradation, sont ajoutés $16 \mu \mathrm{l}$ d'alcool absolu et $4 \mu \mathrm{l}$ de pyridine. Après $30 \mathrm{mn}$ de centrifugation $\left(3600 \mathrm{~g}\right.$ à $\left.4{ }^{\circ} \mathrm{C}\right) 20 \mu \mathrm{l}$ de surnageant sont séchés dans une fiole Wheaton. Quelques heures avant l'analyse, les produits sont sylilés selon la méthode de BROBST \& LOTT (1966) modifiée par DUBERNET (1974). Le tétracosane est utilisé comme étalon interne. Le dosage est effectué avec un chromatographe Packard 437A contenant une colonne en acier de $2,8 \mathrm{~m}$ de long remplie de SE 302 p. 100 sur support chromosorb WHP 80-100 Mesh. Le débit d'azote est de $15 \mathrm{ml} / \mathrm{mn}$. La température initiale est de $100^{\circ} \mathrm{C}$, la température finale $300{ }^{\circ} \mathrm{C}$ avec un programme de montée en température de $10^{\circ} \mathrm{C} / \mathrm{mn}$. Le même chromatogramme per-

\begin{tabular}{|c|c|c|c|c|}
\hline Date des infestations & $3 / 7 / 84$ & $10 / 7 / 84$ & $17 / 7 / 84$ & $24 / 7 / 84$ \\
\hline AOUT & $\begin{array}{l}20 / 8^{*} \\
27 / 8\end{array}$ & $\begin{array}{l}20 / 8^{*} \\
27 / 8\end{array}$ & & \\
\hline SEPTEMBRE & $\begin{array}{r}3 / 9 \\
11 / 9 \\
19 / 9 \\
24 / 9\end{array}$ & $\begin{array}{r}3 / 9 \\
11 / 9 \\
19 / 9 \\
24 / 9\end{array}$ & $\begin{array}{l}3 / 9 * \\
11 / 9 \\
19 / 9 \\
24 / 9\end{array}$ & $\begin{array}{l}3 / 9^{*} \\
11 / 9 \\
19 / 9 \\
24 / 9\end{array}$ \\
\hline OCTOBRE & \multicolumn{4}{|c|}{$\begin{array}{l}\text { pour tous les lots, } \\
\text { prélèvements le : } \\
8 / 10-18 / 10\end{array}$} \\
\hline NOVEMBRE & \multicolumn{4}{|c|}{$15 / 11$} \\
\hline DÉCEMBRE & \multicolumn{4}{|c|}{$12 / 12$} \\
\hline JANVIER & \multicolumn{4}{|c|}{$9 / 1 / 1985$} \\
\hline FÉVRIER & \multicolumn{4}{|c|}{$15 / 2$} \\
\hline MARS & \multicolumn{4}{|c|}{$1 / 3-19 / 3$} \\
\hline AVRIL & \multicolumn{4}{|c|}{$3 / 4-9 / 4-15 / 4-24 / 4$} \\
\hline MAI & \multicolumn{4}{|c|}{$3 / 5-6 / 5-13 / 5-20 / 5-29 / 5$} \\
\hline JUIN & \multicolumn{4}{|c|}{$6 / 6-13 / 6-20 / 6$} \\
\hline
\end{tabular}

Figure 2

Expérimentation Versailles 1984-1985. Dates des 4 infestations artifi cielles en pontes de pyrale et dates des prélevements des chenilles au dernier stade larvaire.

* : Date des premiers prélèvements correspondant à l'apparition des premières larves au dernier stade.

Field trial program, Versailles 1984-1985. Dates of the 4 egg infestations and dates of sampling of last instar larvae.

* : Date of first sampling corresponding to the first last instar larvae.

met d'analyser les concentrations du tréhalose mais aussi la présence d'autres composés glucidiques comme par exemple le glycérol.

\section{Observation des ébauches alaires}

Le prélèvement et l'observation extemporanés des disques imaginaux alaires s'effectue selon la méthode décrite par LAVENSEAU (1976). Rappelons que les 2 ébauches alaires mésothoraciques sont prélevées après incision des pleurites puis portées avec une goutte de Ringer dans une chambre humide d'épaisseur uniforme. Les ébauches alaires dont le développement est rapide et continu chez les larves en développement continu, restent bloquées à un stade peu différencié (au maximum stade III-3) pendant la diapause (fig. 3) (LAVENSEAU \& TRABELSI, 1983).

\section{RÉSULTATS}

\section{A. Origine géographique des pyrales et sortie de dia- pause}

Les résultats correspondent aux observations du printemps 1984 sur 2 populations : Colmar et Versailles. 


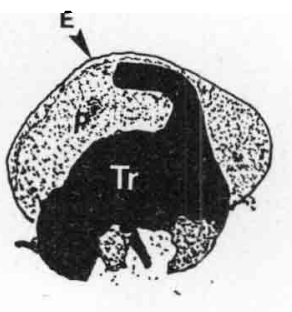

A

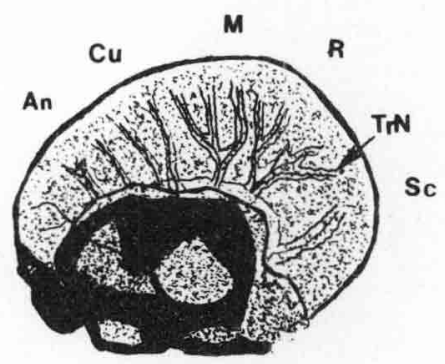

B
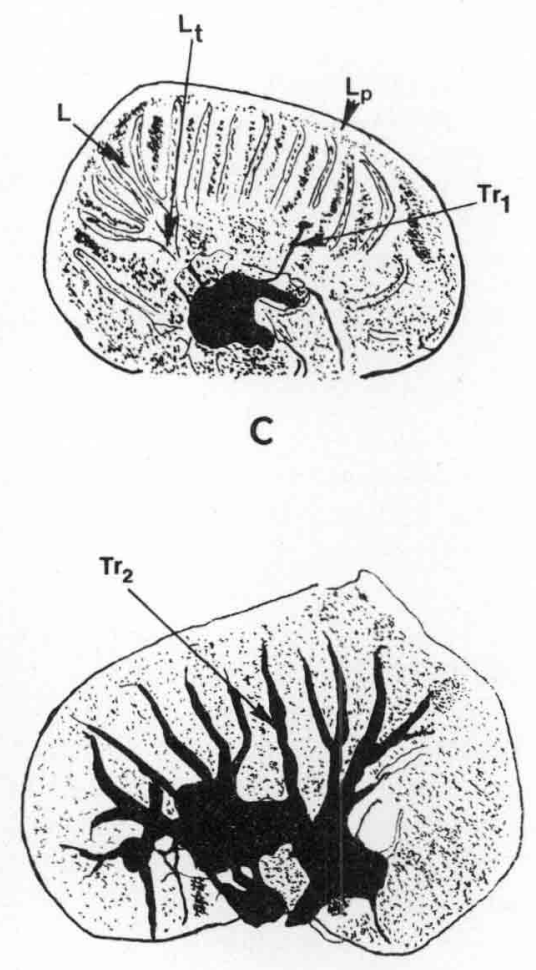

D

Figure 3

Développement des disques imaginaux alaires de la pyrale au cours du $5^{e}$ stade larvaire.

$A:$ stade I, B : stade III-3, C : stade IV, D : stade VI, An : système anal, $C u$ : système cubital, $E$ : enveloppe alaire, $L$ : lacune, $L p$ : lacune périphérique, $L t:$ lacune transversale, $M:$ système médial, $P$ : parenchyme, $R$ : système radial, $S c$ : système subcostal, $T r:$ trachéole, $\operatorname{Tr} 1$ : trachéole primaire, $\operatorname{Tr} 2$ : trachéole secondaire, $\operatorname{Tr} N$ : trachée nymphale.

Development of imaginal wing discs during the last larval instar of the European corn borer.

$A:$ stage $I, B$ : stage $I I I, C$ : stage $I V, D:$ stage VI, An: anal system, $\mathrm{Cu}$ : cubital system, $E$ : peripodial wing sac, $L$ : lacuna, $L p$ : peripheral lacuna, $L t:$ transverse lacuna, $M:$ medial system, $P$ : wing disc parenchyma, $R$ : radial system, $S c:$ subcostal system, $\operatorname{Tr}$ : tracheole, $\operatorname{Tr} 1$ : primary tracheole, $\operatorname{Tr} 2$ : secondary tracheole, $\operatorname{TrN}$ : pupal tracheole.

\section{Critère métabolique}

Au cours de l'hiver, la tréhalosémie est élevée (voisine de $4 \mathrm{~g} / \mathrm{l}$ ) et correspond à celle des larves élevées en photopériode courte en laboratoire (TRABELSI, 1984) (fig. 4). En mai, le taux de tréhalose redevient presque équivalent à celui de larves élevées en conditions de photopériode longue, donc à développement continu. Ces résultats confortent ceux obtenus en laboratoire quand des larves en diapause sont placées en condition de rupture de diapause $\left(18 \mathrm{~L}: 6 \mathrm{D}, 25^{\circ} \mathrm{C}\right.$ avec humidité) (Trabelsi, 1984). Aussi bien à Colmar qu'à Versailles, la tréhalosémie a chuté au cours du mois d'avril, c'est-à-dire 2 mois avant que les prélèvements sur le terrain fournissent 50 p. 100 de nymphes (15 juin). Avec les techniques utilisées, cette diminution de la tréhalosémie est le premier signe visible de la rupture de diapause chez les chenilles.

Dans les 2 populations étudiées, la tréhalosémie est redevenue très importante au mois de mai, très supérieure même à celle de chenilles en diapause. SHIMADA et al. (1984) notent le même phénomène de remontée
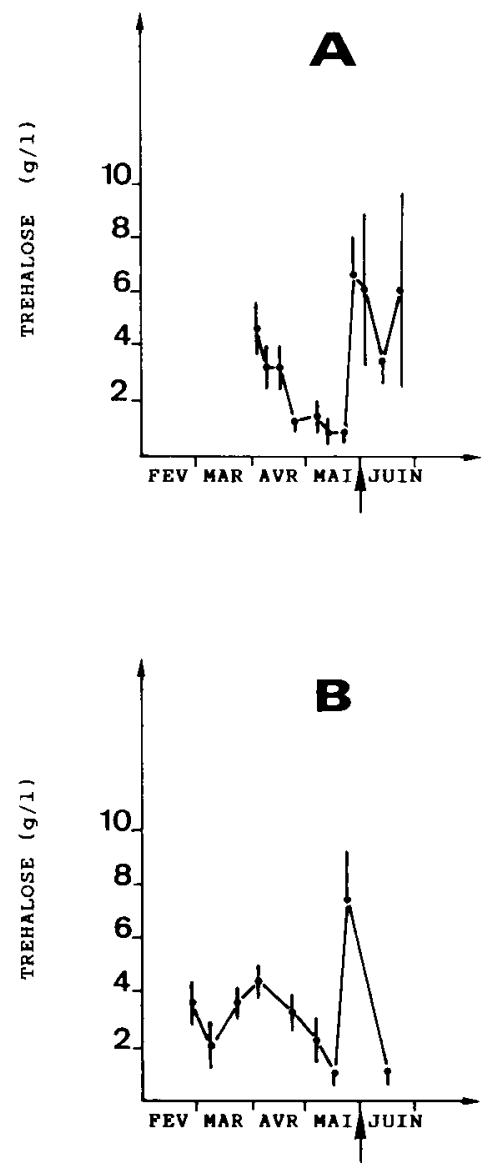

Figure 4

Tréhalosémie de larves de pyrale du maïs issues d'infestations artificielles réalisées à Colmar et Versailles. Printemps 1984. La flèche indique la date de reprise de développement des ébauches alaires. $A=$ larves issues de Versailles, $B=$ larves issues de Colmar $m \pm \operatorname{ESM}(n=10):$ moyenne de 10 valeurs \pm Erreur standard de la moyenne.

Haemolymphatic trehalose concentration of European corn borer larvae obtained from egg infestations at Colmar and Versailles. Spring 1984. The arrow indicates the date of resumption of development of wing discs.

$A=$ larvae from Versailles, $B=$ larvae from Colmar $m \pm S E M$ $(n=10):$ mean of 10 points \pm standard error of the mean. 
du tréhalose juste avant la nymphose chez Leguminivora glycinivorella Matsumura sans toutefois l'expliquer. La variabilité des mesures individuelles, la faible taille des échantillons mesurés et la connaissance insuffisante des facteurs induisant la rupture de diapause ne permettent pas de tirer des conclusions plus formelles, en particulier de savoir si les variations constatées peuvent être expliquées par les seules conditions climatiques.

\section{Critère morphogénétique}

Le développement des disques imaginaux alaires, bloqué à un stade peu avancé pendant la diapause, reprend à partir de la fin du mois de mai, aussi bien chez des larves issues des populations de Versailles que de Colmar. Cette reprise du développement s'effectue un mois après la chute de la tréhalosémie caractéristique de la levée de diapause et 2 semaines avant l'apparition de 50 p. 100 des nymphes dans les prélèvements (15 juin à Versailles et à Colmar) (fig. 4). A titre de comparaison, dans les conditions de laboratoire $(18 \mathrm{~L}$ : $6 \mathrm{D}, 25^{\circ} \mathrm{C}$ ), le développement des ébauches alaires reprend 2 jours après la chute de la tréhalosémie et 7 jours avant l'apparition des premières nymphes.

\section{B. Influence de la date du dépôt des pontes sur les dates d'entrée et de sortie de diapause}

Les premiers stades L5, observés par dissection des cannes, apparaissant à la mi-août proviennent des premières parcelles infestées. Les premiers stades L5 issus des 2 dernières infestations se manifestent 15 jours plus tard. Le décalage est donc réduit de moitié par rapport à l'échelonnement du dépôt des pontes au cours du mois de iuillet (fig. 2 et 5).

Pour les 2 dernières infestations, la durée de la vie larvaire, avant le dernier stade, est donc réduite par rapport aux 2 premières infestations.

Ce «télescopage » du développement larvaire se retrouve au cours de l'été 1985 (fig. 6).

\section{Entrée en diapause}

\section{a) Critère métabolique}

Les chenilles issues d'infestations échelonnées pendant le mois de juillet 1984 deviennent hypertréhalosémiques à peu près au même moment malgré les décalages de départ (fig. 5). La tréhalosémie commence à devenir importante à partir de la mi-octobre (température voisine de $10^{\circ} \mathrm{C}$, fig. 1), mais il faut attendre le mois de novembre (température entre 5 et $10^{\circ} \mathrm{C}$ ) pour noter une hypertréhalosémie élevée de type diapause résultant de l'homogénéisation tardive des populations étudiées. En effet, en août et septembre, l'analyse des taux individuels de tréhalose révèle des différences importantes entre les larves. On est donc vraisemblablement en présence d'un mélange de larves en diapause (fort taux de tréhalose) et de larves en développement continu (faible taux de tréhalose). Ces dernières amorcent une deuxième génération vouée à l'échec, en particulier à cause des conditions climatiques impossibles à supporter sans mécanismes de résistance adaptés. Dans les conditions de laboratoire, le taux de tréhalose de type diapause (supérieur à $5 \mathrm{~g} / \mathrm{l}$ ) apparaît
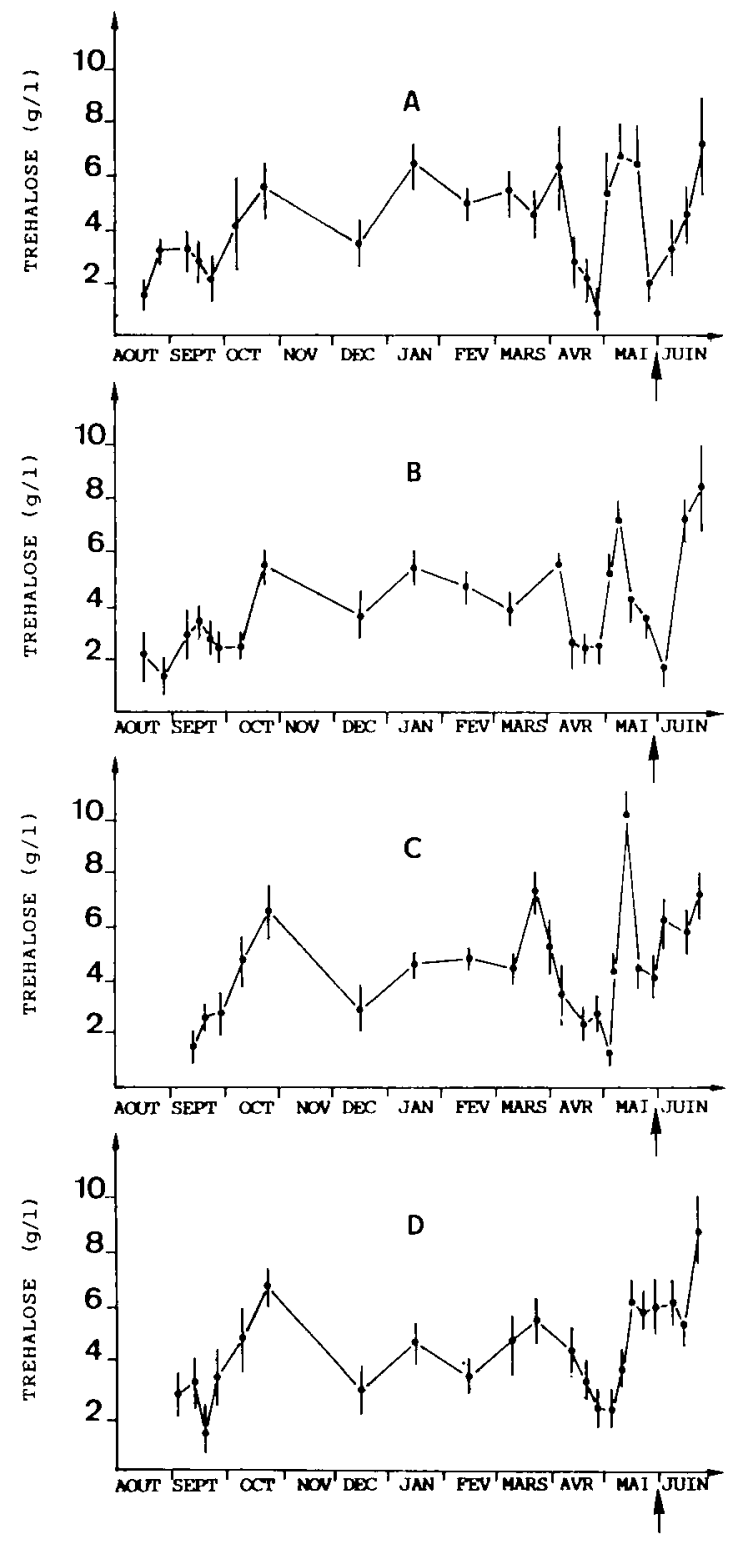

Figure 5

Expérimentation Versailles 1984-1985. Tréhalosémie des larves de pyrale du mais provenant d'infestations artificielles. La flèche indique la date de reprise de développement des ébauches alaires.

Date des infestations : $A=3 / 7 / 84$

$$
\begin{aligned}
& B=10 / 7 / 84 \\
& C=17 / 7 / 84 \\
& D=24 / 7 / 84
\end{aligned}
$$

$m \pm \operatorname{ESM}(n=10)$.

Field trial program, Versailles 1984-1985. Trehalose concentration of European corn borer larvae obtained from egg infestation on corn plant. The arrow indicates the date of resumption of development of wing discs.

Date of egg infestation : $A=3 / 7 / 84$

$$
\begin{aligned}
& B=10 / 7 / 84 \\
& C=17 / 7 / 84
\end{aligned}
$$

$m \pm \operatorname{SEM}(n=10)$

plus tôt : en moyenne après 15 jours de dernier stade larvaire. En effet, les conditions optimum d'élevage assurent une plus grande homogénéité et une plus grande rapidité des développements.

A l'inverse de ce que nous venons de décrire pour 1984, les dosages du tréhalose au cours de l'automne 1985 révèlent des fortes concentrations de sucre dès les premiers prélèvements, au mois d'août (fig. 6) (tempé- 

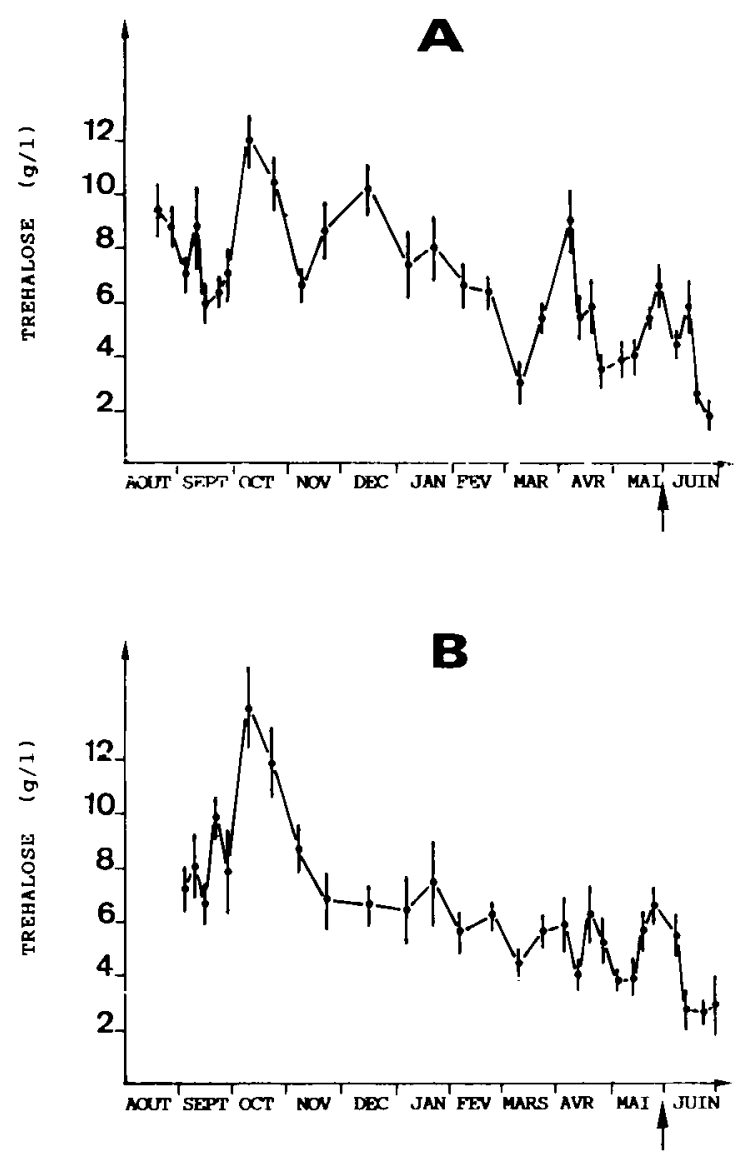

Figure 6

Evolution de la tréhalosémie chez des larves de pyrale de mais, issues d'infestations artificielles. Versailles, 1985-1986. La flèche indique la date de reprise de développement des ébauches alaires.

$A$ : infestation du $4 / 7 / 85$

$B$ : infestation $d u 1 / 8 / 85$

$m \pm \operatorname{ESM}(n=10)$.

Variations of haemolymphatic trehalose concentrations of European corn borer larvae from Versailles, 1985-1986. The arrow indicates the date of resumption of development of wing discs.

$A$ : egg infestation of $4 / 7 / 85$

$B$ : egg infestation of $1 / 8 / 85$

$m \pm \operatorname{SEM}(n=10)$.

rature voisine de $15^{\circ} \mathrm{C}$ ). Simultanément, l'observation des chromatogrammes montre une augmentation de glycérol importante en septembre et en octobre (fig. 7). A partir de cette époque, tréhalose et glycérol existent en forte concentration dans l'hémolymphe.

\section{b) Critère morphogénétique (fig. 8)}

En conditions de laboratoire, LAVENSEAU \& TRABELSI (1983) ont montré que les disques imaginaux alaires ne peuvent dépasser un stade de développement peu avancé (stade III-3, fig. 3) pendant la diapause. Les expérimentations des années 1985 et 1986 montrent que les larves issues des pontes déposées à des périodes différentes ont toutes des disques imaginaux peu développés de type III-1, III-2, III-3 jusqu'à la fin de l'été. Cette observation ne permet donc pas, à elle seule, de déterminer si la chenille est en diapause ou non. Pour l'installation de la diapause, seul le taux de tréhalose est donc utilisable.

Il est néanmoins intéressant de noter que certaines des larves issues des 4 infestations possédaient des disques imaginaux alaires au stade III-3 (30 p. 100). Aucune ébauche alaire de type supérieur à III-3 (fig. 3)
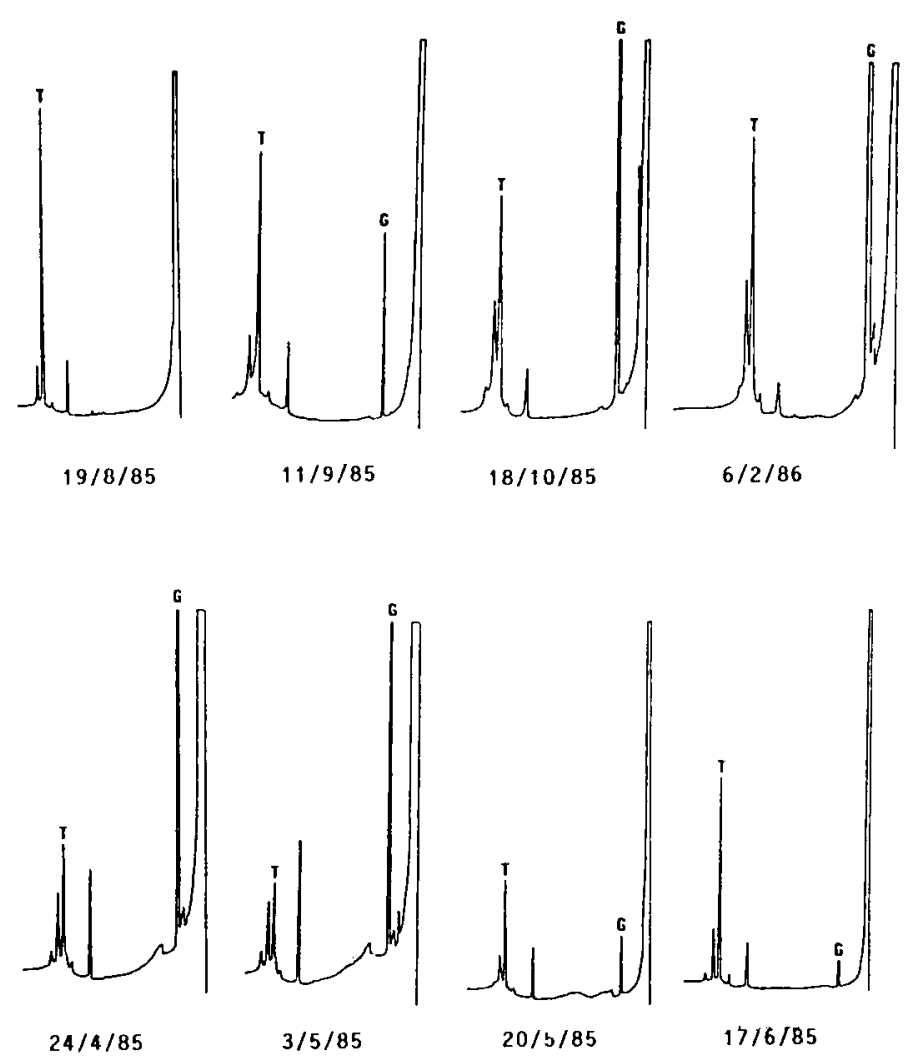

Figure 7

Evolution des sucres de l'hémolymphe d'une population naturelle de pyrales du maïs en diapause (Versailles). Chromatographie en phase gazeuse. $T$ : tréhalose, $G:$ glycérol.

Variations of haemolymphatic carbohydrates of a natural population of European corn borer in diapause (Versailles). Gas chromatography. $T:$ trehalose, $G:$ glycerol.

n'a pu être décelée dans les prélèvements. Bien que rien ne contredise l'imminence d'une diapause, les faibles tréhalosémies correspondantes ont permis de conforter l'hypothèse selon laquelle ces larves ont été prélevées alors qu'elles poursuivaient un développement de type normal.

Ceci est confirmé par la disparition totale des larves ayant des ébauches alaires de type III-3 à partir de la fin du mois d'octobre.

\section{Diapause installée}

\section{a) Critère métabolique}

Pendant tout l'hiver, la tréhalosémie des larves issues des différentes infestations reste importante et correspond au taux de tréhalose observé chez des larves élevées en condition de photopériode courte (fig. 5 et 6). La concentration de glycérol augmente notablement et devient nettement supérieure au taux de tréhalose (fig. 7).

\section{b) Critère morphogénétique}

Chez toutes les larves, le développement des disques imaginaux alaires est arrêté au stade III-1-III-2 pendant l'hiver. Néanmoins, une faible évolution du développement des ébauches est perceptible, celles-ci passant du stade III-1 au stade III-2 (fig. 8). PEYPELUT (résultat non publié) a pu montrer que cette lente évolution matérialisait partiellement la notion théorique de développement de diapause formulée par ANDRE- 


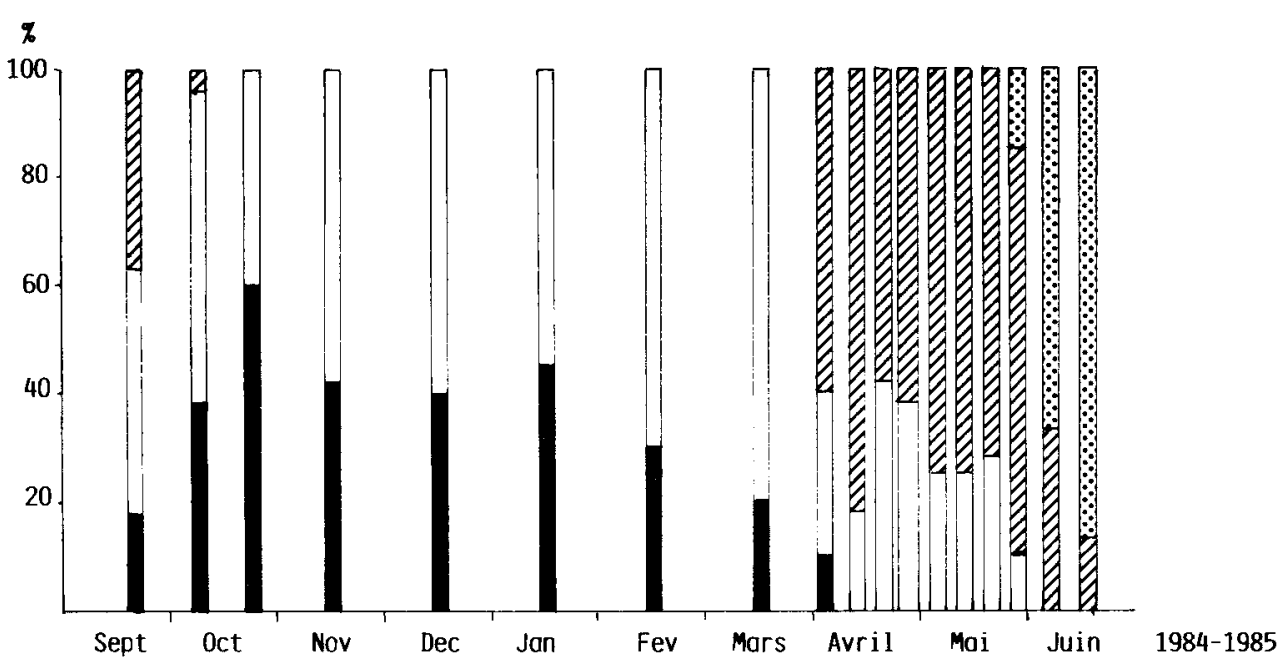

Figure 8

Expérimentation Versailles 1984-1985. Evolution des ébauches alaires des larves de pyrale du maïs provenant d'infestations artificielles (somme des 4 lots réunis).
: stade $111-1$
$\square$ : stade $I I I-2$
ZZI : stade III-3
Q $:$ slade $>I I I-3$

WARTHA (1952). L'apparition du stade de blocage III-3 a lieu à partir du mois d'avril, lorsque la température moyenne commence à s'élever. Les mécanismes d'action des facteurs climatiques sur le développement des ébauches ne sont cependant pas suffisamment connus pour pouvoir les modéliser. D'autres études en conditions de laboratoire sont nécessaires. L'utilisation d'un modèle de degrés-jours ne serait pas adapté car, durant ces périodes, les températures sont proches de la base de degrés-jours. Dans ces conditions, l'hypothèse de linéarité entre la vitesse de développement et la température, sur laquelle repose ce modèle, n'est plus vérifiée. A la fin du mois de mai, toutes les larves ont atteint le stade de blocage III-3 (fig. 8).

\section{Levée de diapause}

\section{a) Critère métabolique}

La tréhalosémie chute à partir de la fin du mois d'avril simultanément chez toutes les larves, issues pourtant d'infestations artificielles décalées dans le temps. Les mêmes résultats sont obtenus en 1985. Le phénomène est beaucoup plus atténué en 1986 (fig. 5 et 6).

Le taux de glycérol diminue brutalement au début du mois de mai, donc plus tardivement que celui du tréhalose.

$\mathrm{Au}$ mois de mai, les concentrations de tréhalose redeviennent très élevées alors que celles du glycérol restent irrémédiablement faibles dans les conditions naturelles (fig. 7).

\section{b) Critère morphogénétique}

A la fin du mois de mai, toutes les larves ont des disques imaginaux alaires dont le développement correspond au stade III-3. La levée de la diapause se manifeste en même temps chez toutes les larves provenant des différentes infestations. Elle se caractérise par la reprise du développement des ébauches alaires selon
Field trial program, Versailles, 1984-1985. Development of wing discs from European corn borer larvae obtained from egg infestations (sum of the 4 infestations).

: stage III-I

: stage $I I I-2$

ZZZ : stage III-3

603 : stage $>I I I-3$

l'échelle des stades définie par LAVENSEAU \& T'RABELSI (1983) (fig. 5 et 6). Du point de vue du critère morphologique, les larves ont donc levé leur diapause.

Les premiers prélèvements révélant un taux de nymphose de $50 \mathrm{p} .100$ sont apparus vers la mi-juin dans les 2 expérimentations consécutives, donc 2 mois après la chute du tréhalose observée en avril et 2 semaines après la reprise du développement des ébauches alaires.

\section{DISCUSSION ET CONCLUSION}

LAVENSEAU \& TRABELSI (1983) ont caractérisé l'état de diapause, en conditions de laboratoire, en utilisant plusieurs critères dont un critère morphogénétique (disques imaginaux alaires). Les résultats que nous venons d'exposer montrent qu'il peut être appliqué à l'étude de certaines des phases de la diapause d'une population naturelle.

Ainsi, l'observation du développement des ébauches alaires permet d'apprécier la progression du développement de diapause. Ce critère d'étude s'est avéré simple à utiliser car il nécessite peu de matériel et permet une visualisation "satisfaisante 》 du déroulement de la diapause. HILAL, dans notre laboratoire, a pu s'y référer pour étudier la diapause de la sésamie (LAVENSEAU et al., 1987).

La reprise du développement des ébauches alaires a lieu 2 semaines avant la nymphose, phénomène habituellement retenu comme révélateur de la levée de diapause.

L'emploi du critère morphogénétique s'avère peu efficace pour apprécier l'entrée en diapause d'un échantillon de larves en fin d'été et au début de l'automne. L'observation d'une ébauche alaire ne donne en effet aucune indication sur son développement à venir. 
Par contre, l'étude de la morphogenèse alaire permet de déterminer quelle fraction de la population observée va continuer son développement et donc disparaître dès le début de l'hiver. En effet, si le stade III-3 a été dépassé, c'est le signe évident d'un développement continu. L'étude de la tréhalosémie confirme que les larves récoltées à Versailles à la fin du mois d'août et du début du mois de septembre sont pour une partie en diapause et pour une autre partie en développement continu (de l'ordre de 30 p. 100 dans la région parisienne).

L'utilisation du seul critère métabolique (tréhalosémie) est plus délicate. En 1984, il avait fallu attendre le début du mois d'octobre pour obtenir un taux caractéristique de diapause. En été 1985, le taux de tréhalose est élevé dès le mois d'août. Le problème qui se pose alors est celui des limites de validité de ce critère pour détecter le début effectif de la diapause. Le taux élevé de tréhalose caractéristique de cette période du cycle biologique de la pyrale du maïs est-il directement lié à la mise en place de la diapause ou bien est-il plutôt induit, chez des larves programmées pour la diapause, par les conditions extérieures? Dans ce cas, les variations chronologiques de mise en place de l'hypertréhalosémie observée sur 2 années consécutives reflèteraient plus les différences météorologiques subies par la larve que le moment exact où l'on peut considérer la diapause comme définitivement installée.

Au cours des 3 années d'expérimentation, la tréhalosémie des chenilles provenant de Versailles a «chuté » au cours du mois d'avril de façon significative. Notons qu'à cette période de l'année, la photophase n'est que de $13 \mathrm{~h} \mathrm{30}$. Il est classiquement admis (BECK \& HANEC, 1960) que 15 h 30 de lumière, au minimum, sont nécessaires pour lever la diapause de la pyrale. TAUBER \& TAUBER (1976) émettent l'hypothèse que la photopériode agit sur la levée de diapause de 2 façons différentes. Soit la photophase devient plus longue que la photophase critique de maintien de diapause (ce serait, selon BECK \& HANEC, le cas de la pyrale à 15 h 30 de lumière, en conditions de laboratoire), soit le seul allongement de la photophase induit la levée de la diapause. Une seule espèce (Chrysopa carnea) se conformerait à ce deuxième cas de figure (TAUBER \& TAUBER, 1973). Le résultat observé semble montrer que la pyrale réagit également à l'allongement de la photopériode.

En 1984 et 1985, le tréhalose a atteint rapidement des concentrations voisines de celles de larves en développement continu ( $1 \mathrm{~g} / \mathrm{l})$. Au contraire, en 1986, le tréhalose a décru de manière plus progressive, mais sensible, vers des concentrations de l'ordre de $3 \mathrm{~g} / 1$. On ne peut donc pas émettre de règle générale quant à l'utilisation du tréhalose pour la levée de diapause, sur ces 2 essais. Ce critère se révèle quand même utilisable à condition d'effectuer des dosages suivis. En cas de chute brutale de la tréhalosémie, les indications seront très précises. En cas de baisse progressive, les renseignements fournis seront plus difficilement utilisables.

L'évolution comparée de la tréhalosémie des larves, provenant de Versailles et de Colmar, au cours du programme 1984, semble montrer que la levée de diapause intervient à la même période, pour ces 2 populations réputées différentes.
Enfin, pour ce qui concerne les lots issus d'infestations décalées dans le temps, les mesures effectuées en 1985 et en 1986 montrent que le taux de tréhalose chute plus ou moins brutalement à la même période. Il n'y a donc pas de différence nette entre les lots. Or ces larves de pyrale sont parvenues au stade L5 à des dates différentes, et semblent avoir entamé leur diapause de façon échelonnée. Comme le montre l'observation des ébauches alaires, tout se passe comme si le repos hivernal avait homogénéisé cet ensemble de populations différentes pour ne plus en former qu'une seule. Il faut donc envisager que des phénomènes physiologiques importants se déroulent pendant la diapause, phénomènes vraisemblablement très lents mais susceptibles de permettre dans certains cas le rattrapage d'un retard initial. La connaissance d'un tel « développement de diapause », dont l'évolution des ébauches alaires n'est qu'un reflet partiel, est capitale pour l'étude de la dynamique des ravageurs.

Nos résultats font également apparaître la possibilité de réapparition d'une très forte tréhalosémie après la rupture de diapause. Elle pourrait être liée à un phénomène de quiescence que l'on peut provoquer en laboratoire par le froid ou la sécheresse (LAVENSEAU et al., 1985) mais que les conditions climatiques ne permettent pas d'envisager clairement dans les populations étudiées. Pour plus de sûreté, la rupture de diapause doit donc être diagnostiquée en utilisant les deux critères. La chute de la tréhalosémie constitue le premier indice. Ses éventuelles "remontées 》ultérieures indiquent les périodes de quiescence dont il faut tenir compte pour réaliser des prévisions de l'émergence des adultes. En second lieu, le critère morphogénétique (ébauches alaires) est nécessaire pour confirmer les phases de quiescence.

Enfin, nous avons vu que le glycérol peut également, par la baisse pratiquement irréversible de son taux, indiquer que les conditions globales de reprise du développement sont remplies. Dans ce cas, toute hypertréhalosémie ne peut être que passagère et liée à une quiescence. PEYPELUT a bien montré que cette accumulation de glycérol est liée au froid puisqu'on n'observe pas sa présence chez des chenilles en diapause conservées à $25^{\circ} \mathrm{C}$ (résultats non publiés). Nous confirmons l'hypothèse que, pour la pyrale (BARNES \& HODSON, 1956 ; ASAHINA, 1966) comme pour d'autres espèces (ASAHINA, 1969 ; DANKS, 1978 ; SALT, 1959), le glycérol est, avec le tréhalose, une molécule importante pour lutter contre le gel hivernal. Quand le taux de glycérol chute irrémédiablement au printemps, les hypertréhalosémies occasionnelles observées pourraient être des réponses rapides de l'animal pour résister aux périodes de froid tardif ou pour lutter contre la déshydratation.

Cette étude a donc montré que l'emploi de critères physiologiques apporte des précisions utiles pour distinguer les différentes phases de la diapause et analyser la dynamique des populations naturelles de pyrale du maîs. La bonne compréhension de cette période longue et relativement mal connue du point de vue physiologique est actuellement indispensable pour pouvoir améliorer les modèles de prévision de la date d'infestation. Utilisables en conditions de laboratoire comme de plein champ, les critères présentés permettront d'estimer et de $t$ - ter ces modèles dans les 2 types de condi- 
tions. Ils constituent donc un outil de qualité pour assurer la construction et la fiabilité d'un modèle.

Reçu le 24 novembre 1986.
Accepté le 30 juillet 1987.

\section{REMERCIEMENTS}

Nous remercions Monsieur M. Stengel (I.N.R.A. Zoologie de Colmar) pour la fourniture des larves de pyrale, et Monsieur P. ANGiLADE pour son analyse critique du manuscrit.

\section{RÉFÉRENCES BIBLIOGRAPHIQUES}

Andrewartha H. G., 1952. Diapause in relation to the ecology of insects. Biol. Rev., 27, 50-107.

Asahina E., 1966. Freezing and frost resistance in insects. In Cryobiology (Ed. by Meriman H. T.), Academic Press, New York, 451-486.

Asahina E., 1969. Frost resistance in insects. Adv. Insect Physiol., 6, 1-50.

Asahina E., Tanno K., 1964. A large amount of trehalose in a frost resistant insect. Nature, 204, 1222.

Barnes D., Hodson A. C., 1956. Low temperature tolerance of the European corn borer in relation to winter survival in Minnesota. $J$. econ. Entomol., 49, 19-24.

Beck S. D., Hanec S. W., 1960. Diapause in the European corn borer, Ostrinia nubilalis. J. Insect Physiol., 4, 304-318.

Brobst K. M., Lott C. E., 1966. Determination of some components in corn syrup by gas-liquid chromatography of the trimethyl-silyl derivatives. Cereal Chem., 43, 35.

Danks H. V., 1978. Modes of seasonal adaptation in the insects. I. Winter survival. Can. Entomol., 110, 1167-1205.

Dubernet M. O., 1974. Application de la chromatographie en phase gazeuse à l'étude des sucres et des polyols du vin. Thèse $3^{\mathrm{e}}$ cycle, Bordeaux, $148 \mathrm{p}$.

Got B., 1985. Approche de la modélisation de la dynamique de population de la pyrale du maïs (Ostrinia nubilalis $H b n$.) (Lepidoptera : Pyralidae) dans le Bassin parisien. Thèse $3^{e}$ cycle, Paris VII, $210 \mathrm{p}$.

Hawlitzky N., 1986. Etude de la biologie de la pyrale du maïs, Ostrinia nubilalis Hbn. (Lep. Pyralidae) en région parisienne durant 4 années et recherche d'éléments prévisionnels du début de ponte. Acta Oecologica, Oecol. Appl., 7 (1), 47-68.

Hayakawa Y., Chino H., 1981. Temperature-dependent conversion between glycogen and trehalose in diapausing pupae of Philosamia cynthia and pryeri. Insect Biochem., 11, 43-47.
Lavenseau L., 1976. Etude descriptive et expérimentale du développement post-embryonnaire des ébauches alaires de quelques Lépidoptères. Thèse Doct. Sci. Nat., Bordeaux I, 221 p.

Lavenseau L., Trabelsi M., 1983. Le développement des disques imaginaux alaires chez la pyrale du mais, critère organique de diapause ou non diapause. Agronomie, 3, 523-528.

Lavenseau L., Peypelut L., Gadenne C., Anglade P., 1985. Facteurs externes et diapause chez la pyrale du maïs, Ostrinia nubilalis $\mathrm{Hbn}$. (Lép., Pyralidae). Bull. Soc. Zool. Fr., 210 (3), 275-279.

Lavenseau L., Gadenne C., Hilal A., Peypelut L., 1987. The endocrine control of diapause in insects. In "Advances in Invertebrate Reproduction Vol. 4" (Ed. Porchet, Andries, Dhainaut), Elsevier Sci. Publ., Amsterdam, 69-78.

McLeod D. G. R., 1976. Geographical variation of diapause termination in the European corn borer, Ostrinia nubilalis (Lepidoptera: Pyralidae) in southwestern Ontario. Can. Ent., 108, 1403-1408.

Moreau R., 1969. Observations sur les taux de tréhalose au cours du développement direct et de la diapause chez Pieris brassicae. $C . R$. Acad. Sci., Paris, 268, 1441-1444.

Salt R. W., 1959. Role of glycerol in the cold-hardenirg of Bracon cephi (Gahan). Can. J. Zool.. 37. 59-69.

Shimada K., Sakagami S. F., Honma K., Tsutsui H., 1984. Seasonal changes of glycogen/trehalose contents, supercooling points and survival rate in mature larvae of the overwintering soybean pod borer Leguminivora glycinivorella. J. Insect. Physiol., 30, 369-373.

Stengel M., 1982. Essai de mise au point de la prévision des dégâts pour la lutte contre la pyrale du maïs (Ostrinia nubilalis) en Alsace (Est de la France). Entomophaga, 27, 105-114.

Tauber M. J., Tauber C. A., 1973. Seasonal regulation of dormancy in Chrysopa carnea (Neuroptera). J. Insect Physiol., 19, 1455-1463.

Tauber M. J., Tauber C. A., 1976. Insect seasonality : diapause maintenance, termination and postdiapause development. Ann. Rev. Entomol., 21, 81-107.

Trabelsi M., 1984. Caractérisation de la diapause chez la pyrale du mais. Thèse $3^{\mathrm{e}}$ cycle, Bordeaux I, $84 \mathrm{p}$. 